\title{
Resección de la tibia proximal con reconstrucción endoprotésica y del mecanismo extensor de rodilla en paciente con osteosarcoma
}

\author{
Proximal resection of the tibia with endoprosthetic \\ and extensor knee mechanism reconstruction \\ in a patient with osteosarcoma
}

Luis Tinoco Téllez, * Martín Espinosa Castolo, ${ }^{\neq}$ Ernesto Marín y Santillán, $\$$ Ramiro Gálvez Valdovinos, $\$$ Juan Francisco Funes Rodríguez, $\$$ Luis Gerardo Domínguez Carrillo

Citar como: Tinoco TL, Espinosa CM, Marín SE, Gálvez VR, Funes RJF, Domínguez CLG. Resección de la tibia proximal con reconstrucción endoprotésica y del mecanismo extensor de rodilla en paciente con osteosarcoma. Acta Med GA. 2021; 19 (4): 539-543. https://dx.doi.org/10.35366/102542

\section{Resumen}

Introducción: La resección de la tibia proximal con reconstrucción endoprotésica es un procedimiento complejo de conservación de la extremidad en presencia de tumores de la tibia proximal. Avances recientes en implantes y en las técnicas quirúrgicas han hecho que esta opción sea preferible a la amputación para muchos pacientes. La supervivencia a largo plazo y las tasas de recurrencia son similares entre el salvamento y la ablación de la extremidad. Caso clínico: Femenino de 55 años con dolor de seis meses de evolución en tercio superior de pierna izquierda a $2 \mathrm{~cm}$ por debajo de tuberosidad tibial y aumento de volumen en dicha zona, con estudios de imagen y biopsia se confirma osteosarcoma, sin metástasis; se realiza resección de la tibia proximal y reconstrucción endoprotésica. Conclusiones: La resección de la tibia proximal con reconstrucción protésica puede estar asociada con complicaciones significativas; sin embargo, éstas pueden minimizarse con planificación preoperatoria adecuada y una técnica meticulosa.

Palabras clave: Tumor óseo, resección, reconstrucción de la tibia proximal, endoprótesis.

\section{Abstract}

Introduction: The proximal resection of the tibia with endoprosthetic reconstruction is a complex limb preservation procedure in the presence of tumors of the proximal tibia. Recent advances in implants and surgical techniques have made this option preferable to amputation for many patients. Long-term survival and recurrence rates are similar between limb salvage and limb ablation. Case report: A 55-year-old woman with the pain of six months of evolution in the upper third of the left leg to $2 \mathrm{~cm}$ below the tibial tuberosity and increased volume in this area, with imaging and biopsy studies, confirmed osteosarcoma, without metastasis; proximal tibial resection and endoprosthetic reconstruction were performed. Conclusions: The proximal resection of the tibia with prosthetic reconstruction may be associated with significant complications; however, these can be minimized with proper preoperative planning and meticulous technique.

Keywords: Bone tumor, resection, proximal tibial reconstruction, endoprosthesis.
* Especialista en Cirugía Oncológica. División de Cirugía del Hospital Angeles León.

₹ Ortopedista. Cirugía articular. División de Cirugía de UMAE Bajío.

$\S$ Cirujano General. División de Cirugía del Hospital Angeles León.

" Especialista en Medicina de Rehabilitación. Profesor de la Facultad de Medicina de León. Universidad de Guanajuato.

Hospital Angeles León. León, Guanajuato. México.
Correspondencia:

Dr. Luis Gerardo Domínguez Carrillo

Correo electrónico: Igdominguez@hotmail.com

Aceptado: 11-11-2020.

www.medigraphic.com/actamedica

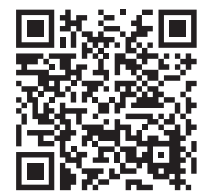




\section{INTRODUCCIÓN}

A la tibia proximal corresponde el segundo sitio anatómico más común en la presencia de sarcomas óseos primarios (de 19 a 80\%) con una incidencia de 400 casos/año. ${ }^{1}$ Históricamente el procedimiento de elección para lesiones óseas malignas en esta área fue la amputación (por arriba o a través de la rodilla), siendo esta modalidad de tratamiento la recomendada debido a la dificultades asociadas con el salvamento de la extremidad así como la recurrencia al no efectuar la resección radical. ${ }^{2}$ En los últimos 20 años, los avances en técnicas quirúrgicas y terapia adyuvante permiten el salvamento de la extremidad con la resección amplia de sarcomas óseos primarios sin afectar los cinco años de tasas de supervivencia. Actualmente, las opciones quirúrgicas para las lesiones en la tibia proximal implican tanto la amputación como la preservación de la extremidad; las primeras incluyen la amputación o plastia de rotación; las segundas incluyen la artrodesis con autoinjerto o aloinjerto, el reemplazo de la tibia proximal y la reconstrucción con aloinjerto, el uso de aloinjerto protésico, y la endoprótesis. Los reportes oncológicos han demostrado que la cirugía de preservación de la extremidad aunada a terapia adyuvante no aumenta la mortalidad al compararla con los procedimientos ablativos, e incluso pueden llegar a incrementar la supervivencia a cinco años así como disminuir la recurrencia. ${ }^{3}$ Sin embargo, uno de los inconvenientes de la cirugía de preservación de la extremidad implica mayor morbilidad tanto en el perioperatorio como en el postoperatorio. La reconstrucción endoprotésica tiene una tasa de complicación temprana de $22 \%$ que aumenta a 55\% con el seguimiento prolongado. ${ }^{4}$ Con reconstrucciones de aloinjerto los resultados buenos o excelentes sólo se logran en $43 \%$ de pacientes debido al alto número de complicaciones postoperatorias. Algunos pacientes preferirán la amputación, ya que permite una actividad sin restricciones y menos posibles complicaciones. Otros desean la reconstrucción de la extremidad para obtener resultados cosméticos más aceptables así como menor gasto energético que cuando se usa una prótesis externa. El uso de endoprótesis localizadas en la tibia proximal implica dificultades técnicas derivadas de la distorsión y resección de la anatomía local así como de la reconstrucción del mecanismo extensor de la rodilla. A lo anterior debe agregarse la presencia de complicaciones como la inadecuada cobertura por los tejidos blandos y las alteraciones neurovasculares por la íntima relación de la tibia proximal con los vasos y nervios relacionados. ${ }^{5}$

\section{PRESENTACIÓN DEL CASO}

Femenino de 55 años, sin antecedentes de importancia, quien inició seis meses previos a ser evaluada, con dolor en tercio superior de pierna izquierda a $2 \mathrm{~cm}$ por debajo de tuberosidad tibial, siendo de mayor intensidad durante la ambulación, el cual disminuía con uso de paracetamol. Acude a consulta por notar aumento de volumen en dicha zona, se solicitaron estudios de imagen, los cuales revelaron tumor en tercio proximal de la tibia. Se le hospitalizó para realizar estudios con el fin de complementar el diagnóstico, las radiografías mostraron lesión de carácter blástico en tercio superior de tibia izquierda (Figura 1), la biopsia confirmó osteosarcoma. Los estudios de laboratorio con resultados normales para citometría hemática, pruebas de funcionamiento hepático, electrólitos y función renal, sólo discreta elevación de deshidrogenasa láctica (340 UI/L) y de fosfatasa alcalina (155 UI/L). Se efectuó tomografía computarizada de tórax con resultados normales, tomografía computarizada y resonancia magnética para planeación quirúrgica así como centellograma óseo corporal total (tecnecio) encontrando sólo afección de tibia izquierda. Se le estadificó como osteosarcoma central convencional intramedular de alto grado de tipo osteoblástico en etapa IIA, T2, N0, M0. La paciente se sometió a resección de la tibia proximal (Figura 2), su reconstrucción con endoprótesis (prótesis tumoral OSS, Stryker. USA) y el mecanismo extensor de rodilla con colgajo de gastrocnemio y cierre de piel utilizando injerto de mediano grosor de piel de muslo ipsilateral (Figuras 3 y 4). Su evolución postoperatoria fue satisfactoria (Figura 5), se refirió a rehabilitación, logrando a los tres meses: flexoextensión activa de rodilla de 0 a $65^{\circ}$, con fuerza muscular de 4 en escala de Daniels para cuádriceps izquierdo y $5 / 5$ para resto de músculos ipsilaterales

\section{Figura 1:}

Radiografía anteroposterior de rodilla y tercio superior de tibia izquierda que muestra lesión blástica localizada a la metáfisis tibial.

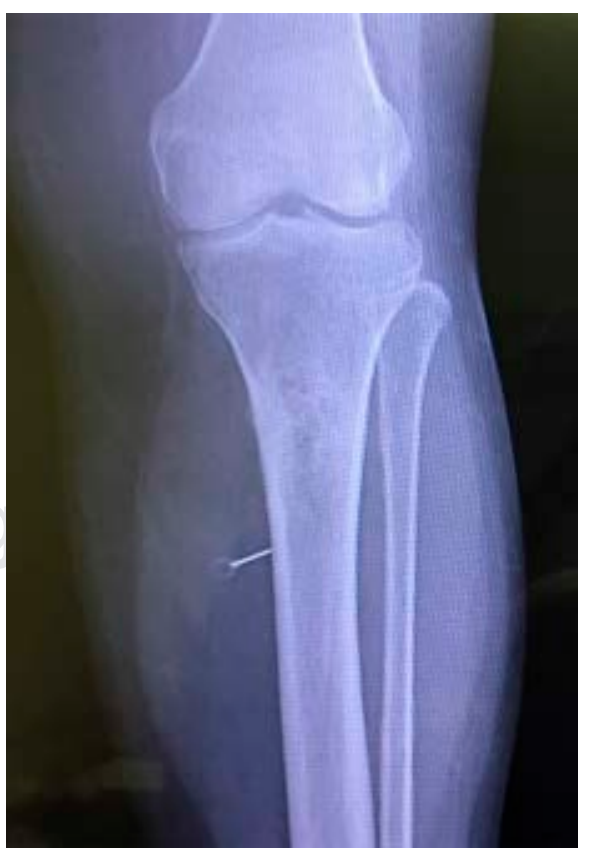




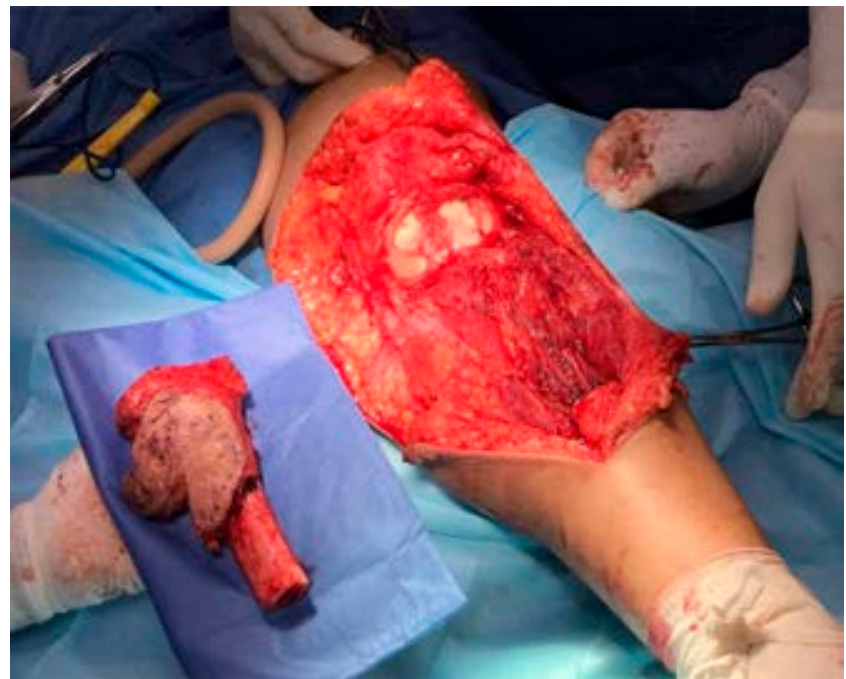

Figura 2: Resección de la tibia proximal izquierda y pieza quirúrgica de osteosarcoma.

y contralaterales; no presentó anormalidades en la sensibilidad, y con llenado capilar normal, con independencia en todas las ADVH. Se anota que fue canalizada a oncología médica desde una semana previa a la cirugía continuando actualmente su control con ifosfamide-etoposide.

\section{DISCUSIÓN}

La resección de sarcomas tibiales proximales con la reconstrucción endoprotésica es potencialmente peligrosa. Los procedimientos de rescate de extremidades están llenos de complicaciones que van desde infección superficial hasta pérdida de la extremidad o la vida, especialmente cuando ésta se realiza en el segmento proximal de la tibia, incluso con los avances actuales en la cobertura de tejidos blandos de componentes protésicos, las tasas de infección se mantienen significativamente altas, siendo de $12 \%$ (previamente eran hasta de 33\%). Las tasas de reoperación y amputación secundaria a 10 años continúan altas, correspondiendo a 70 y $25 \%$, respectivamente. ${ }^{6}$ Sin embargo, los buenos resultados funcionales justifican el salvamento de la extremidad cuando sea posible ante problemas que incluyen: osteosarcoma, histiocitoma fibroso maligno, sarcoma de Ewing y tumor de células gigantes. ${ }^{7}$ m

Las contraindicaciones relativas a la resección de la tibia proximal y la reconstrucción endoprotésica incluyen infiltración directa del tumor a la trifurcación poplítea, fractura patológica, la necesidad de resección de la tibia de más de dos tercios de su longitud, biopsia mal ubicada, y sepsis local o sistémica. Aunque las prótesis expandibles ya están disponibles, la edad temprana en el momento del diagnóstico limita las opciones de reconstrucción si el crecimiento residual previsto es significativo. ${ }^{8}$

Además de una evaluación sistémica completa para detectar lesiones metastásicas y repercusiones sistémicas de la enfermedad, es de especial importancia la valoración de pulsos distales así como la sensibilidad en los dermatomas del pie y pierna, la evaluación motora en la rodilla, el tobillo y pie, ya que una extremidad inferior avascular, pérdida de sensibilidad y la penetración del tumor a través de la piel son contraindicaciones a los procedimientos de salvamento de la extremidad.

Los estudios de imagen deben incluir radiografías simples y resonancia magnética de la rodilla y tibia entera para evaluar el tejido óseo y los tejidos blandos Si el fémur distal o las estructuras intraarticulares están involucrados, entonces la resección extraarticular de la rodilla es requerida. El nivel de resección debe ser de 3 a 5 cm más allá del área

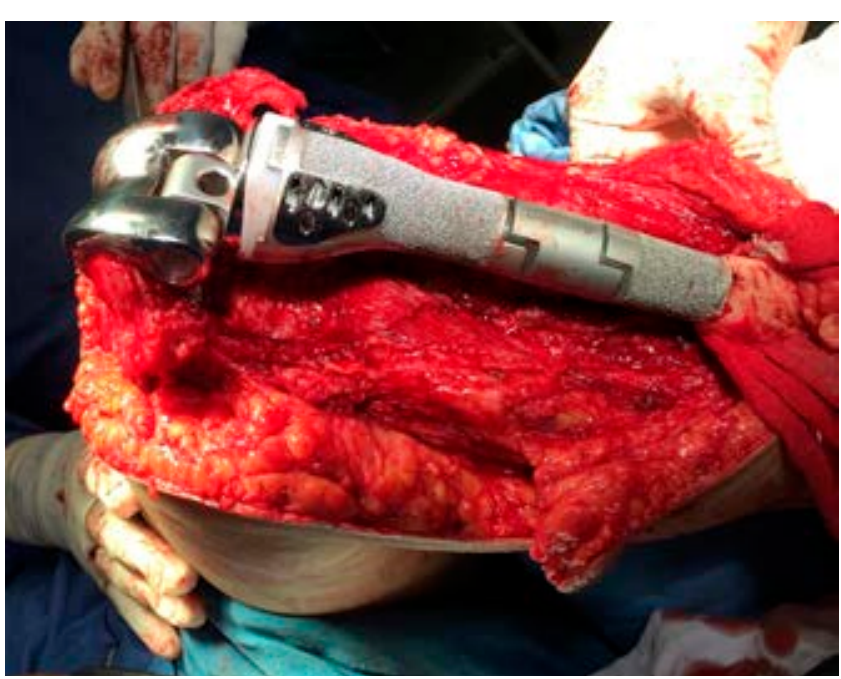

Figura 3: Reconstrucción con endoprótesis de tibia proximal y rodilla izquierda.

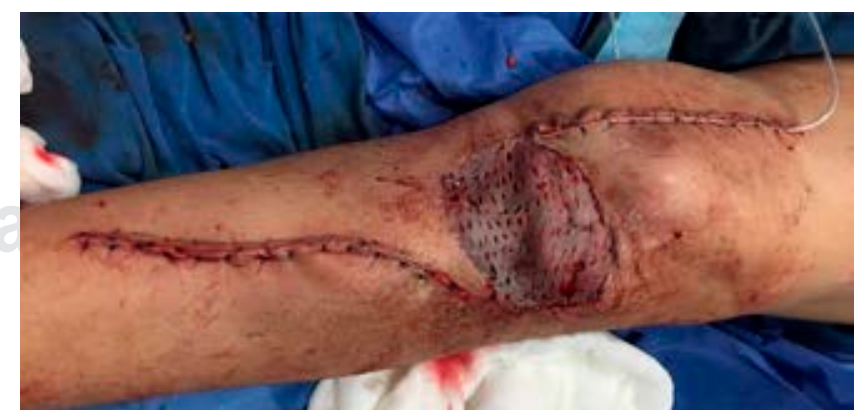

Figura 4: Cierre de incisión quirúrgica y cobertura con injerto de piel tras reconstrucción de mecanismo extensor de rodilla con colgajo de gastrocnemio medial. 

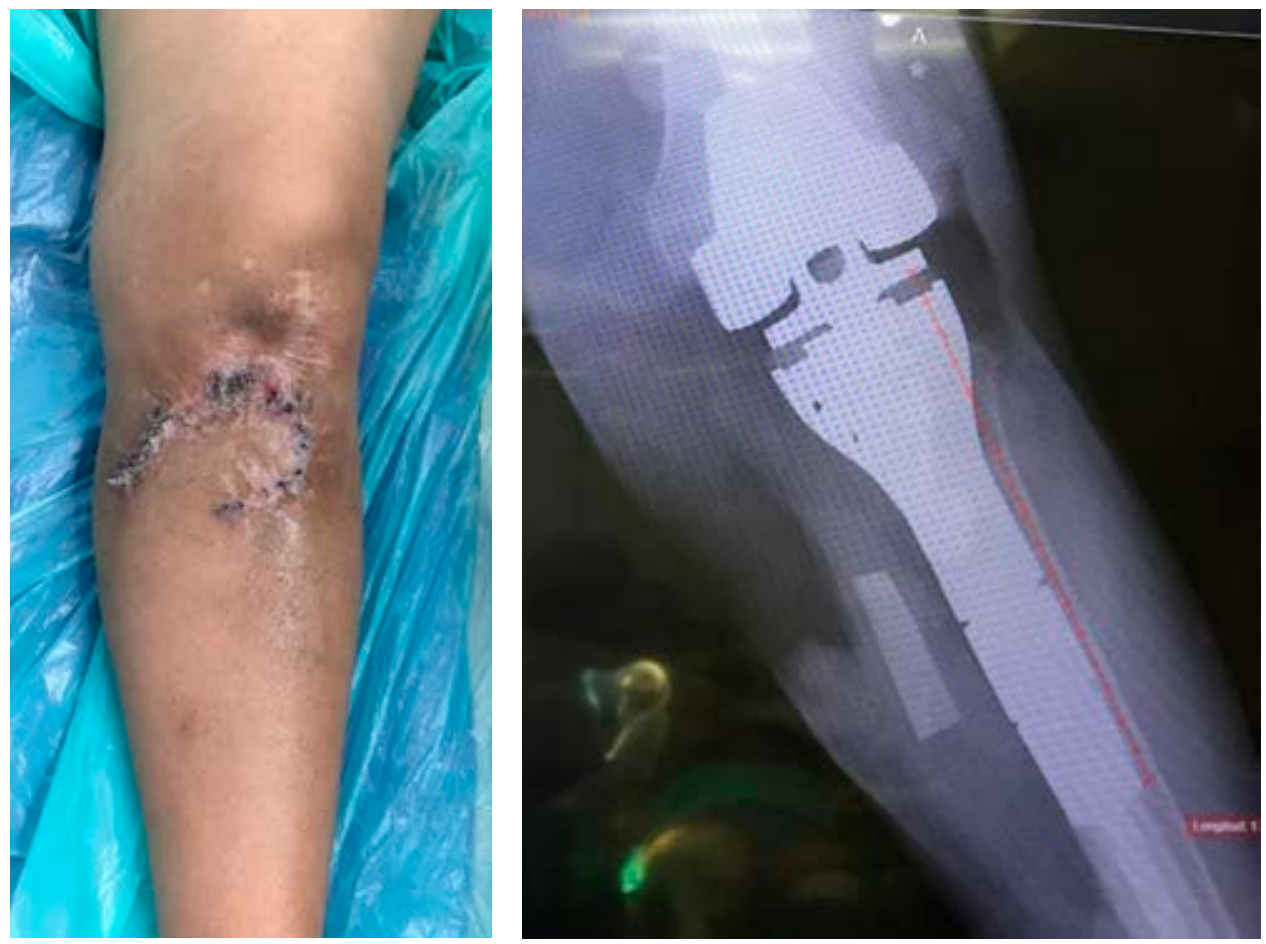

Figura 5:

Cuatro semanas postcirugía, estado de cicatrización y control radiográfíco.

afectada por la lesión y a su vez permitir el salvamento del tercio distal tibial. Si la resonancia magnética demuestra una extensión tumoral posterior hacia los vasos poplíteos, es necesario realizar angiografía para la evaluación de la bifurcación de la arteria poplítea, ya que el tronco tibioperoneo permite llevar sangre al pie. Los angiogramas en vista lateral permiten valorar la viabilidad entre el borde posterior del tumor y los vasos poplíteos, además se indica la combinación de un escáner óseo y la tomografía computarizada de tórax para evaluar la presencia de metástasis. El equipo de trabajo debe incluir al oncólogo médico o pediátrico y al oncólogo radioterapeuta, pues ambos proporcionan la información más actualizada y la atención adyuvante efectiva que puede aumentar significativamente la supervivencia del paciente y la disminución de la recurrencia. ${ }^{1-3}$

En general, una reconstrucción exitosa de la tibia proximal y su reconstrucción con endoprótesis consta de tres tiempos: a) la resección del tumor, que incluye desde la incisión hasta el mantenimiento de los vasos poplíteos y los nervios, b) la reconstrucción de la tibia proximal y la articulación de la rodilla con una componente protésico modular y, c) la reconstrucción del mecanismo extensor funcional de la rodilla y su cobertura tisular; cada etapa implica dificultades técnicas y complicaciones inherentes. ${ }^{9}$

La implementación de técnicas quirúrgicas depuradas y la planeación multidisciplinaria han mejorado significativamente el resultado de la reconstrucción endoprotésica en la resección de tumores de la tibia proximal, las tasas de recurrencia y supervivencia equivalentes han promovido a la cirugía de salvamento de la extremidad sobre los procedimientos ablativos. Actualmente, el concepto de la resección de la tibia proximal es relativamente uniforme entre los cirujanos, ${ }^{10}$ no así las técnicas de reconstrucción de la tibia proximal y la articulación de la rodilla y más aún la reconstrucción del mecanismo extensor con cobertura de tejidos blandos en la que aún existe controversia entre los cirujanos, ya que algunos utilizan aloinjerto osteoarticular, mientras que otros prefieren la endoprótesis. Al respecto, la reconstrucción con aloinjerto ha mostrado resultados ligeramente inferiores tanto en funcionalidad como en las tasas de supervivencia a cinco años; ${ }^{11,12}$ también se han usado implantes compuestos por aloinjerto-prótesis para reconstruir la articulación de la rodilla, ${ }^{13}$ observando que tanto las tasas de infección como la falla del mecanismo extensor son más altas con este tipo de reconstrucción (ambas con $23 \%$ de manera respectiva). ${ }^{2}$ Por otra parte, se ha descrito la reconstrucción del mecanismo extensor sin utilizar colgajo del gastrocnemio, conectando el tendón rotuliano directamente a la prótesis; sin embargo, la extensión efectiva de rodilla es insuficiente, e implica reintervención con tasas de $10 \%$. Por otro lado, de acuerdo a los reportes el uso de colgajo del gastrocnemio para la reconstrucción del mecanismo extensor de rodilla con una endoprótesis ha proporcionado los resultados más 
confiables y predecibles en comparación con las otras opciones reconstructivas. ${ }^{14}$

\section{CONCLUSIONES}

La resección de la tibia proximal con reconstrucción endoprotésica es un procedimiento complejo de conservación de la extremidad en presencia de tumores de la tibia proximal, los avances recientes en implantes y en las técnicas quirúrgicas han hecho que esta opción sea preferible a la amputación para muchos pacientes.

\section{REFERENCIAS}

1. National Comprehensive Cancer Network. NCCN Clinical Practice Guidelines in Oncology. Bone Cancer. Version 1.2017. NCCN. Available in: https: //www.nccn. org/professionals/physician gls/pdf/bone.pdf

2. Song WS, Kong CB, Jeon DG, Cho WH, Kim MS, Lee JA et al. Prognosis of extremity osteosarcoma in patients aged 40-60 years: a cohort/case controlled study at a single institute. Eur J Surg Oncol. 2010; 36 (5): 483-488.

3. Strauss SJ, Frezza AM, Abecassis N, Bajpai J, Bauer S, Biagini R et al. Bone sarcomas: ESMO Clinical Practice Guidelines for diagnosis, treatment and follow-up. Ann Oncol. 2014; 25 (Suppl)3: 113-123.

4. Adle Z, Robert, Quinn HR. Proximal tibial resection and prosthetic reconstruction. Techniques in Orthopaedics. 2007; 22: 1-11. doi: 10.1097/BTO.0b013e31811eb507.

5. Mavrogenis FA, Angelin A, Pala E, Sakellariou IV, Ruggiere P, Papagelopoulos JP. Reconstruction of the extensor mechanism after major knee resection. Orthopedics. 2012; 35: e672-680.
6. Biau D, Faure F, Katsahian S, Jeanrot C, Tomeno B, Anract P. Survival of total knee replacement with a megaprosthesis after bone tumor resection. J Bone Joint Surg Am. 2006; 88 (6): 1285-1293.

7. Ek EW, Rozen WM, Ek ET, Rudiger HA. Surgical options for reconstruction of the extensor mechanism of the knee after limbsparing sarcoma surgery: an evidence-based review. Arch Orthop Trauma Surg. 2011; 131 (4): 487-495.

8. Gorlick R, Janeway K, Marina N. Osteosarcoma. In: Pizzo PA, Poplack DG, Adamson PC, Blaney SM, Helman LJ. Principles and practice of pediatric oncology. 7th ed. Philadelphia: Wolters Kluwer; 2016, 876-897.

9. Wittig JC, Villalobos CE, Hayden BL, Choi I, Silverman AM, Malawer M. Osteosarcoma of the proximal tibia: limb-sparing resection and reconstruction with a modular segmental proximal tibia tumor prosthesis. Ann Surg Oncol. 2010; 17 (11): 3021. doi: 10.1245/ s10434-010-1180-3.

10. Prabowo Y, Primaputra AR, Kodrat E. Reconstruction of osteosarcoma of the proximal tibia using bone on polyethylene hemiarthroplasty knee joint system: a case report. Int J Surg Case Report. 2020; 72: 188-196.

11. Smolle AM, Andreou D, Tunn UP, Leithner A. Advances in tumour endoprostheses: a systematic review. Oncology. 2019; 4: 445-459. doi: org/10.1302/2058-5241.4.180081.

12. Chim H, Tan BK, Tan MH, Tan KC, Song C. Optimizing the use of local muscle flaps for knee megaprosthesis coverage. Ann Plast Surg. 2007; 59: 398-403.

13. Mattei JC, Curvale G, Rochwerger A. Surgery in "around the knee" bone tumors. Bull Cancer. 2014; 101 (6): 571-579. doi: 10.1684/ bdc.2014.1983.

14. Ottaviani G, Robert RS, Huh WW, Jaffe N. Functional, psychosocial and professional outcomes in long-term survivors of lower-extremity osteosarcomas: amputation versus limb salvage. Cancer Treat Res. 2010; 152: 421-436. 